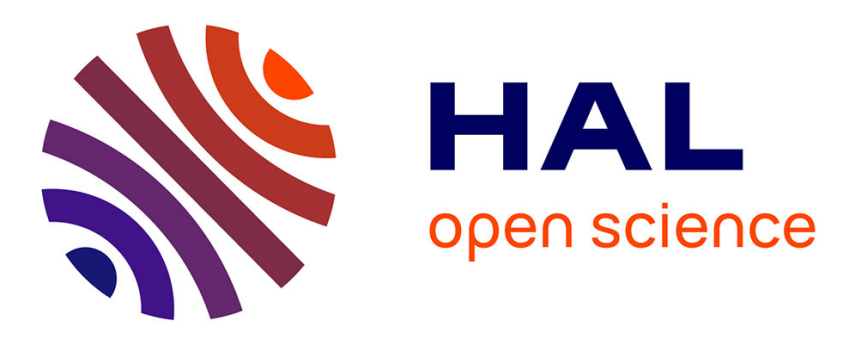

\title{
Indiens dans la ville, bourgeois dans la jungle : l'Alliance bolviarienne (ALBA) enlisée sur le front climatique
}

Fabrice Andréani

\section{- To cite this version:}

Fabrice Andréani. Indiens dans la ville, bourgeois dans la jungle : l'Alliance bolviarienne (ALBA) enlisée sur le front climatique. Mouvements: des idées et des luttes, 2012, 70 (2), pp.59. 10.3917/mouv.070.0059 . halshs-02022903

\section{HAL Id: halshs-02022903 \\ https://shs.hal.science/halshs-02022903}

Submitted on 18 Feb 2019

HAL is a multi-disciplinary open access archive for the deposit and dissemination of scientific research documents, whether they are published or not. The documents may come from teaching and research institutions in France or abroad, or from public or private research centers.
L'archive ouverte pluridisciplinaire HAL, est destinée au dépôt et à la diffusion de documents scientifiques de niveau recherche, publiés ou non, émanant des établissements d'enseignement et de recherche français ou étrangers, des laboratoires publics ou privés. 


\title{
INDIENS DANS LA VILLE, BOURGEOIS DANS LA JUNGLE : L'ALBA ENLISÉE SUR LE FRONT CLIMATIQUE
}

\author{
Fabrice Andreani \\ La Découverte | Mouvements
}

\author{
$2012 / 2-n^{\circ} 70$ \\ pages 59 à 69
}

ISSN 1291-6412

Article disponible en ligne à l'adresse:

http://www.cairn.info/revue-mouvements-2012-2-page-59.htm

Pour citer cet article :

Andreani Fabrice, « Indiens dans la ville, bourgeois dans la jungle : l'ALBA enlisée sur le front climatique », Mouvements, 2012/2 n 70, p. 59-69. DOI : 10.3917/mouv.070.0059

Distribution électronique Cairn.info pour La Découverte.

(c) La Découverte. Tous droits réservés pour tous pays.

La reproduction ou représentation de cet article, notamment par photocopie, n'est autorisée que dans les limites des conditions générales d'utilisation du site ou, le cas échéant, des conditions générales de la licence souscrite par votre établissement. Toute autre reproduction ou représentation, en tout ou partie, sous quelque forme et de quelque manière que ce soit, est interdite sauf accord préalable et écrit de l'éditeur, en dehors des cas prévus par la législation en vigueur en France. II est précisé que son stockage dans une base de données est également interdit. 


\section{Indiens dans la ville, bourgeois dans la jungle : l'ALBA enlisée sur le front climatique}

Le sommet de Copenhague aurait pu marquer un tournant dans les négociations sur le changement climatique. Elles avaient peu de chances d'aboutir, mais la mobilisation de la société civile laissait en effet entrevoir de l'espoir : celui de construire un rapport de force contraignant les pays industrialisés à prendre des mesures drastiques de réduction de leurs émissions de gaz à effet de serre. Les pays de l'ALBA ont tenté de proposer des débouchés officiels aux revendications des militants de la justice climatique. La Bolivie convoquait une rencontre inédite, de militants écologistes et altermondialistes, de représentants des peuples indigènes, de négociateurs, d'experts et de chefs d'État, au premier trimestre 2010. Elle a débouché sur l'adoption d'un ambitieux " accord des peuples ", dont le contenu a été soumis par la Bolivie à l'Onu. Mais l'alliance bolivarienne semble depuis avoir renoncé à le défendre.

Pour le comprendre, une analyse des principes structurants la diplomatie bolivarienne est nécessaire.

A u sortir de la COP15 de Copenhague (2009), le bébé géopolitique de Hugo Chávez et Fidel Castro, l'Alliance bolivarienne pour les peuples de Notre Amérique (ALBA-TCP ${ }^{1}$ ), semblait déterminée à relever le niveau des négociations climatiques, en y appuyant les revendications altermondialistes. "Rendez-vous raté " étant donné l'urgence de l'adoption d'accords contraignants, le premier "sommet de la dernière chance pour sauver la planète " d'une longue série a ainsi été le théâtre d'un coup de poker aussi spectaculaire qu'improbable. Accusés de "sabotage " par la presse dominante, les Présidents vénézuélien et bolivien, porte-parole d'une alliance décidée à faire sienne les critiques et slogans émanant du Klima Forum, ont été massivement applaudis tant à leurs tribunes officielles que lors de leur visite éclair aux manifestants,

Par Fabrice Andreani *
* Doctorant, CERI-I.E.P., Paris ; Groupe d'Études Interdisciplinaires sur le Venezuela.

1. "Alternative " puis à la fois "Alliance " et "Traité de commerce des peuples " depuis l'adhésion bolivienne, elle intègre aussi l'Équateur, le Nicaragua, Antigua-et-Barbuda, La Dominique, SaintVincent-et-lesGrenadines. 
2. H. Do Alto et P. STEFANONI, Nous serons des millions. Evo Morales et la gauche au pouvoir en Bolivie, Raisons d'agir, Paris, 2008.

3. L. Boltanski, De la critique. Précis de sociologie de l'émancipation, Gallimard, Paris, 2009. et leurs discours diffusés en boucle sur l'ensemble de la toile alternative. Dans la foulée, ils invitaient des membres de l'intransigeante Climate Justice Now ! (CJN!) à travailler aux côtés du Climate Action Network comme de négociateurs du Nord et du Sud à l'élaboration de revendications communes pour la COP 16 de Cancún (2010). Le Sommet des Peuples sur le changement climatique et les droits de la Terre Mère s'ouvraient dans la mythique province bolivienne de Cochabamba - celle-là même où Evo Morales, alors syndicaliste cocalero, avait remporté quelques années plus tôt la "guerre de l'eau ".

Deux ans plus tard, l'ambitieux Accord de Tikipaya, après avoir circulé dans les espaces alternatifs des quatre coins du monde avec le concours des ambassades de l'ALBA, est resté lettre morte. Ne parvenant à l'imposer comme document officiel à l'Onu, et après avoir dénoncé l'isolement dans lequel l'avaient laissée ses pairs de l'ALBA à Cancún - et même menacé de saisir la Cour pénale internationale pour transgression de l'unanimité requise -, la Bolivie s'est faite à son tour bien plus discrète à Durban (2011). Si Rio +20 sera sûrement prétexte à de nouveaux appels du pied de l'ALBA en direction de l'altermondialisme, ceux qui escomptent encore ne pas désespérer Billancourt sont largement marginalisés au sein du " mouvement des mouvements ". Et pour cause : d'apparence contre-hégémonique, leur discours aux accents téléologiques s'avère, à l'épreuve des faits, porteur des mêmes tares que la rhétorique d'un "Empire " rendu responsable de tous leurs errements. Pour reprendre à notre compte le registre de la paraphrase d'un " grand Karl Marx " cité tous azimuts par Chávez, la diplomatie climatique de l'ALBA nous invite alors à "remettre la dialectique bolivarienne sur ses pieds". Car tout prévisible qu'eût été ce dénouement, elle n'en recèle pas moins une énigme de taille : comment, en connaissance de cause, les plus gros propriétaires de pétrole et de lithium au monde - en sus du gaz à l'échelle latino-américaine - peuvent-ils sérieusement prétendre s'ériger en avantgarde d'une révolution éco-socialiste globale?

\section{- En attendant l'Empire...}

Ce "décalage entre la réalité telle qu'elle est et la réalité telle qu'elle devrait être ${ }^{3}$ " n'est pas spécifique à l'ALBA, il constitue une règle d'or en matière de politique institutionnelle. Mais il atteint ici un tel degré que la superbe avec laquelle les " socialistes du XXIe siècle " s'arrogent une compétence écologique universelle révèle quelque chose de bien plus profond que le cynisme de rigueur dans l'exercice banal de fonctions politiques. Ce qui distingue l'ALBA des autres blocs géopolitiques émanant du "Global South ", c'est bien la croyance de ses classes dirigeantes les plus riches dans le caractère messianique de leur tâche. Plus que tout énoncé sur la "démocratie participative " et éventuellement " protagonique " - et ses déclinaisons juridiques au Venezuela, en Bolivie ou en Équateur -, rien ne résume mieux cet épistémé que l'expression déclinée par l'ancien guérillero et sociologue Alvaro Garcia Linera, actuel vice-président bolivien, dans un article réflexif publié dans Le Monde Diplomati- 
que en septembre 2011 - "Les quatre contradictions de notre révolution " : il y décrit sa coalition de gauche comme un "gouvernement des mouvements sociaux". Les leaders des grands États de l'ALBA s'estiment les héritiers légitimes des guérillas urbaines et paysannes, réseaux clandestins de liaison entre ces dernières et des groupes d'" officier libres ", sinon des mouvements étudiants, ouvriers ou multi-sectoriels, avec l'appui desquels ils ont brigué le pouvoir. L'oxymore en appelle ici un autre, qui est son corollaire extra-national : la doctrine de la "diplomatie des peuples " élaborée à Caracas, au service d'une "intégration " d'un type nouveau, appelée à se réaliser en " cercles concentriques ", des " communautés de base " jusqu'aux institutions supra-nationales : l'État doit mâttriser l'usage juridique des secondes afin de mettre à disposition des premières les ressources nécessaires à leur " développement endogène " - capitaux, infrastructures et savoirs (partage et transferts de technologies), débouchées éventuelles ${ }^{4}$. Pour d'aucuns, " une fois cette dynamique lancée les gouvernements ne devraient même plus jouer le rôle d'intermédiaires entre leurs citoyens respectifs ", l'ALBA assurant dès lors la " mise en réseau des mouvements sociaux $"$.

Or l'art de repousser la révolution effective à un horizon plus lointain, qui est le propre des gouvernants auto-dénommés "révolutionnaires ", se nourrit au quotidien de l'amalgame entre politiques intérieure et extérieure, injonction participative et discipline anti-impérialiste. Ce qui permet ici tour à tour d'excuser l'autoritarisme ponctuel du gouvernement cubain, plongé dans une économie de guerre du fait de l'embargo nord-américain - comme les errements judiciaires des néo-sandinistes nicaraguayens, peu dotés en "avantages comparatifs " miniers - ; et de gager l'approfondissement des procédures démocratiques au Venezuela, en Bolivie, et en Équateur, sur la "souveraineté des ressources stratégiques " récemment - quoique partiellement - nationalisées. De fait, l'ensemble des réalisations au palmarès de l'ALBA repose sur la générosité des États riches en mines polluantes, pour faire vivre à n'importe quel coût la Patria Grande rêvée en son temps par le Libertador Simon Bolivar - avant d'être trahi par "l'oligarchie". Le succès de l'envoi de carburant et le renforcement des capacités de raffinage dans l'ensemble de la Caraïbe, avec des facilités de remboursement par voie de crédits à taux bas ou de troc alimentaire, expliquent que la liste des candidats à l'adhésion s'allonge: Sainte-Lucie, le Surinam, sont désormais invités " spéciaux ", et - last but not least-, Haïti, point de départ historique de la vague d'émancipation nationale sud-américaine, "invité permanent ". Le Venezuela a été déclaré " libre d'alphabétisation " par l'Unesco, et le système de troc de pétrole vénézuélien contre médecins et enseignants cubains, à l'origine de l'ALBA, a fait recette en
4. A. Bansart, $L a$ diplomacia de los pueblos, Cuadernos Nueva Diplomacia, MPPRE, Caracas, 2008.

5. Entretiens, Université bolivarienne du Venezuela, Centre d'études politiques \& Centre d'économie marxiste, juillet 2010 . 
Bolivie et au Nicaragua. Ses alliés peuvent dès lors eux aussi se targuer d'avoir les moyens de remplir les objectifs du millénium avant les États pauvres d'Afrique ou d'Asie. Ajoutée à deux décennies de néolibéralisme néo-autoritaire, la façon dont les franges les plus réactionnaires des bourgeoisies locales perçoivent ces changements, non sans les encouragements d'un South Comandmant Nord-américain toujours prompt à remplir sa " destinée manifeste " dans son "arrière-cour ", donne à ce qui apparaît de facto comme un réformisme " progressiste " une légitimité révolutionnaire : si Correa a pu exagérer la portée d'un coup policier rapidement contrecarré, Morales a bel et bien déjoué au moins un complot orchestré par les secteurs réactionnaires de l'armée et les latifundistas sécessionnistes de la Media Luna. Quant à Chávez, il a le charisme d'un président restitué par la rue suite à un coup d'État de deux jours appuyé par un blackout médiatique total - nonobstant quelques révérences envers le patronat.

L'histoire semble donc se répé-

"Si le climat était une banque, ils l'auraient déjà sauvée "; " changez le système, pas le climat." ter, et quelques mois avant la conférence de Copenhague, ce discours en devient quasi-performatif lorsqu'en juin 2009 le hondurien Manuel Zelaya se fait débouter par un coup d'État à peine quelques jours après son adhésion à l'ALBA. Victimes avérées de l'impérialisme Nord-américain, ceux qui sont devenus des chefs d'États au péril de leur vie se rendent à Copenhague sur le pied de guerre, à la fois "anticapitaliste " et " anti-impérialiste "; mais il est plus juste de traduire ces deux termes par "progressiste " et " anti-Nord-américain ". Le récit autorisé de l'événement fondateur de l'ALBA, à savoir l' " enterrement " de la Zone de libre-échange des Amériques (ZLEA) au sommet de Mar del Plata (2005) illustre bien la nuance : il écarte systématiquement le rôle des intérêts du capitalisme brésilien dans l'échec du projet Nord-américain, au profit de la seule convergence de vues entre l'Alliance sociale transcontinentale et l'ALBA alors en gestation. À la COP15, le schéma interprétatif est le même : le seul véritable "impérialisme " est celui des États-Unis et de leurs alliés, et les États du "Sud " et les mouvements aux inclinaisons anticapitalistes doivent donc s'entendre. Chávez, ex-putschiste de gauche devenu Comandante d'une "révolution démocratique ", porte ici la double-casquette du procureur à charge contre un "système " en pleine crise financière, et du missionnaire philanthrope administrant à un public ignorant sinon "lâche " une leçon d'humanisme et de droit international. Il dénonce, en se payant le luxe de se faire décompter la remarque de son temps de parole, le "document top secret ", symptomatique de la "dictature impériale mondiale " du détenteur de la " machine à fabriquer des billets ", et qui " ne veut pas d'accord " parce qu'" entre le fort et le faible, la liberté opprime ", alors que "seule la loi libère ". Quant à Evo Morales, en premier président indio d'un État désormais " plurinational " et qui a constitutionnalisé le "buen vivir" en lieu et place du "vivir mejor", il 
invoque la "culture de la vie " contre celle " de la "souverains" mort " qui menace d'en finir avec la Pachamama, et dénonce un " génocide " programmé de l'Afrique.

Ces discours n'ont pas simplement pour fonction de troubler la bienséance des assemblées onusiennes, ils sont de bout à bout calibrés de sorte à raisonner au-dehors de celles-ci, leurs vrais destinataires se situant dans les rues. Ainsi Chávez, contempteur du "fantôme qui parcourt les rues de Copenhague ", - ce "capitalisme que personne n'ose nommer ", ne manque pas de saluer ceux qu'il identifie à juste titre comme ses fossoyeurs potentiels, ces "jeunes " qui battent le pavé, "préoccupés à juste titre " des négociations, déplorant qu'il " il n’y a pas suffisamment de place pour les accueillir ". Après avoir pris le soin d'assurer avec Morales aux occupants de la rue que la répression qu'ils ont subie n'existe plus dans leurs territoires " souverains ", il ironisera même dans l'enceinte officielle, sur le traitement " décent " par les " agents de l'ordre " de ceux qui sont parvenus à en braver les contrôles à l'entrée. Et il veille à prendre le temps de leur "rendre hommage "de façon solennelle, en y relayant les deux slogans phares de la mobilisation, qu'il dit publiquement l'avoir le plus touché : "si le climat était une banque, ils l'auraient déjà sauvée "; " changez le système, pas le climat ". En outre, rappelant qu' " il n'y a rien à attendre " de ces discussions feutrées, Morales préconisera tout bonnement d'imposer ce cap en contournant le cadre onusien, ni plus ni moins à travers un "référendum mondial sur le changement climatique "où les " citoyens du monde " seront appelés à juger par eux-mêmes de la "responsabilité du capitalisme " dans les dommages irréversibles infligés à la Pachamama; et de l'urgence de faire passer le réchauffement climatique sous la barre des $2^{\circ} \mathrm{C}$, voire $1,5^{\circ} \mathrm{C}$ (contre 4 selon les scénarios les plus répandus). Mais en posant des objectifs concrètement irréalisables, de l'aveu même des mouvements sociaux, ces vindictes sonnent aussi comme un aveu de faiblesse. Si cette surenchère anti-systémique n'est pas pour déplaire à ses porte-parole comme leurs soutiens inconditionnels, la logique d'accumulation qu'ils prétendent subvertir tout en en tirant l'essentiel de leurs ressources comporte entre-temps des coûts non seulement environnementaux, mais aussi humains.

\section{-...cachez ces barricades que je ne saurais voir !}

Tous ceux qui prendront le temps et les moyens de se déplacer quelques semaines plus tard à Cochabamba n'en attendent pas tant, et il est clair qu'ils s'y rendent en connaissance de cause. Tandis que Chávez et Morales espèrent récolter lors de leur Sommet des peuples les fruits de leurs prestations scéniques et du travail de leurs scribes, nombre de militants escomptent quant à eux en profiter de l'occasion pour mettre l'ALBA devant ses contradictions. Les hôtes donnent de toute façon d'emblée le La, posant comme condition sine qua non à la signature d'un texte commun toute mention, ni même comme objectif à atteindre, de la possibilité d'un abandon des politiques néo-extractivistes. Leur renvoyant la balle, et en vertu d'un rapport de forces défavorable aux gouvernants étant don- 
6. "Pablo Solón : Occupy is a source of hope ", OneClimate, 28 novembre/2011.

7. R. Cavagnoud, "La Bolivie et la révolte des indigènes du Tipnis ", OPALC, octobre 2011. née l'absence des nombreux autres chefs d'État, notamment du G77, attendus pour l'occasion, ils tiennent bon devant les visées hégémoniques de l'ALBA : Morales voit son bilan écologique ouvertement critiqué après qu'on lui ait imposé la tenue d'une dix-huitième table de travail spécifiquement destinée à cet effet ; et se trouve obligé de revenir, du moins formellement, sur l'adoption des mécanismes REDD promus par la Banque mondiale. Surtout, Chávez échoue une fois plus à convaincre de la nécessité d'une Ve Internationale sous la houlette de l'ALBA - contre notamment l'avis de Via Campesina. À Durban, Pablo Solón, ex-ambassadeur bolivien à l'Onu, dira d'ailleurs lui aussi " ne rien attendre " des gouvernements ${ }^{6}$ et miser plutôt sur l'option Occupy.

De fait, la carte institutionnelle apparaît d'autant moins jouable que les conflits sur les territoires des principaux bailleurs de l'ALBA sont légion, et en nombre croissants. Le tableau s'avère ici bien éloigné tant des discours des franges réactionnaires de l'opposition que de l'idéalisme prorégime: ils n'ont aucun rapport avec les "cadeaux aux pauvres " des grandes métropoles de l'ALBA ou des petites États caribéens, pas plus qu'ils ne se limiteraient à des carences écologiques à la campagne ou en forêt conçues comme "prix à payer " d'améliorations sociales en ville. Déjà, à leur retour de Cochabamba, certains participants n'ont pas manqué de rapporter leur étonnement une fois sur place au vu des manifestations publiques fustigeant la gestion environnementale de Morales. Aujourd'hui, en sus des revendications récurrentes d'un syndicalisme mineur influent, Morales doit composer avec les intérêts divergents d'autres composantes du Movimiento al Socialismo (MAS), à l'instar du conflit autour du changement éventuel de la législation protégeant le parc national Tipnis : le noyau historique du MAS, celui des paysans cultivateurs de coca - dont il est issu - exigent au même titre que d'autres communautés dépendant des débouchées extra-locales, des infrastructures routières facilitant les flux de marchandises vers la capitale; tandis que les communautés " indigènes " habitant le parc et qui peuvent encore s'en passer s'y opposent radicalement ${ }^{7}$. Par ailleurs, à Copenhague, Evo n'avait pas manqué de ménager la Chine, dans la mesure même où son gouvernement poursuivait déjà des négociations avec ses diplomates et hommes d'affaires au sujet de l'exploitation du litihum.

Côté vénézuélien, aussi pouvait-on déjà percevoir, en creux dans le discours de Copenhague, les limites à peine voilées de la conception quelque peu étroite que se fait le gouvernement de l'anti-impérialisme, et partant de l'anti-capitalisme. Chávez " n'offense ni ne craint " de critiquer un Barack Obama, qui " a reçu le prix Nobel de la paix le (...) jour où il envoyait 30000 soldats (...) tuer des innocents en Afghanistan "; précisément parce que pour un bon quart, c'est avec ce qui est redevenu, à la faveur des nationalisations partielles, "le pétrole de tous les vénézuéliens ", et qui assure encore $90 \%$ des recettes d'exportation et $30 \%$ du PIB, que sa machine de guerre continue de tourner. De même, ce n'est pas un hasard si le Comandante relaie là explicitement, aux côtés de celle de la délégation soudanaise qui préside alors le G77, les positions chinoise et brési- 
lienne. Eu égard à la première, il prend le soin de rappeler que l'" on ne peut pas [lui] demander (...) la même chose qu'aux États-Unis ", que la liste des dettes contractées à son égard, et remboursées rubis sur l'ongle en pétrole, s'allonge de jour en jour. Quant au Brésil, l'appui de son industrie agro-alimentaire à la "semonce de la rente pétrolière " dans la quête de la "souveraineté alimentaire " justifie le silence sur sa promotion indiscriminée des bio-carburants - que l'affichage pro-régime en interne n’hésite pourtant pas à dénoncer comme " mortifères ". Or si le nouvel "État magique $^{8}$ " peint aux couleurs chavistes a bien pu octroyer aux habitants des barrios des conditions " dignes " de logement, de santé et d'éducation, c'est grâce à la multiplication par 20 du prix du brut en 10 ans ; et au coinvestissement de ces bénéfices dans des entreprises " socialistes " où des multinationales brésiliennes et chinoises sont la plupart du temps, sous différentes formes, associées à la pétrolière PDVSA. Or malgré des améliorations matérielles substantielles, seul le secteur informel échappe aujourd'hui à la véritable guerre de classes qui sévit ailleurs. Ce non sans la complicité tacite de gouverneurs ou de maires du Parti socialiste uni du Venezuela (PSUV) peu zélés dans la lutte contre l'impunité : entre transnationales minières et communautés indigènes, propriétaires terriens et paysans, mais aussi entreprises nationalisées ou partenaires de l'État et ouvriers réclamant une autogestion effective, les tirs croisés sont quotidiens et les morts fréquents, jusque dans les indigènes, ouvriers ou paysans du " chavisme critique "9. Sans parler des coûts écologiques - et partant humains irréversibles de la sur-exploitation pétrolière en Amazonie, attestés récemment par un incident des plus graves de l'histoire pétrolière, comparé ni plus ni moins à celui de BP dans le Golfe du Mexique ${ }^{10}$.

Enfin, si entre Copenhague et Rio, la campagne internationale des diplomates équatoriens pour la récolte de fonds destinés à financer la non-exploitation du pétrole du parc Yasuni n'a pas cessé de faire du bruit, peu sont encore optimistes quant à son issue. Le consentement répété de Rafael Correa à repousser la "date butoir " de la relance de l'exploitation ne compense pas le fait que l'existence même de cette échéance, pour beaucoup inconcevable au vu du statut constitutionnel initial du parc, indique que les jeux sont déjà faits. Malgré l'annonce de l'arrivée tardive de sommes importantes, le projet phare du socialismo del sumak kawsay semble largement compromis ; et ne continuer à exister qu'à l'international, comme vitrine écologique à moindre frais de la coalition AlianzaPaís. Aussi, celle-ci a perdu l'appui d'une partie non négligeable de la Confédération des Associations indigènes équatoriennes, qui l'avait portée au pouvoir. Bien qu'il soit clair que des secteurs réactionnaires voient la dissidence de cette organisation d'un bon oil, et que certains des cadres de la CONAIE puissent succomber à leurs sirènes - à leurs pétrodollars -, il est par trop pratique de l'accuser en bloc d'“ impérialisme ". On comprend également que le naturel avec lequel le président s'est fait dédommager en millions de dollars pour un cas - avéré - de diffamation à son encontre par un quotidien d'opposition, dont il a assisté personnellement au procès, puisse refroidir une partie des soutiens au représentant officiel du
8. F. CORONIL, The magical State. Nature, money and modernity in Venezuela, University of Chicago Press, Chicago, 1997.

9. R. UZCÁTEGUi, La revolución cómo espectaculo. Una crítica anarquista al gobierno bolivariano, Org. Nelson Garrido, Caracas, 2010.

10. www.mediapart. fr/journal/ international/080312/ une-catastropheécologique-embarrassehugo-chavez 
bien vivir à l'équatorienne. Dernier arrivé au pouvoir, Correa semble être celui des dirigeants néo-bolivariens qui a tourné le dos le plus rapidement à la radicalité des ambitions qu'il affichait lors de son investiture.

\section{- La diplomatie contre la révolution ?}

Défendre le protocole de Kyoto, demander la reconnaissance de la "dette écologique " des grandes puissances occidentales, et de sa solvabilité par voie de transferts de "technologies vertes " et de prise en charge

\section{Tout pbénomène de "circulation} internationale des idées " repose sur des stratégies inscrites dans des espaces politiques distincts mais ponctuellement en contact en debors de leurs frontières. des "réfugiés climatiques " venus de territoires en voie de désertification ou inondés : c'est ce qui reste concrètement à l'ALBA comme carte à jouer pour tenter de se racheter à l'occasion de Rio +20 . De façon significative, il s'agit là des points sur lesquels l'ensemble des participants au Sommet des peuples de Cochabamba étaient a minima d'accord avant même la tenue de la conférence. Entretemps, la donne environnementale au sein de l'ALBA s'est aggravée, la crise financière et les soubresauts afférents des prix des matières premières aidant. Mais l'essentiel n'est pas là : au vu de la qualité des oppositions partisanes, en matière environnementale comme sociale, les gouvernants de l'ALBA semblent à ce stade toujours représenter un " moindre mal " dans le cadre de la démocratie légale. Donné son caractère éminemment global, la cause de la " justice climatique " s'avère ainsi une ressource pratique de légitimation face à la démultiplication de ces conflits, puisqu'elle permet de pointer du doigt la responsabilité d'une colonisation pluriséculaire dans le saccage de "Mère Nature ". Renvoyées ainsi à une historicité a-temporelle, les fameuses "bases matérielles " des économies des États primo exportateurs en deviennent naturalisées. Et de fait, si on n'évoque ce capitalisme réellement existant qu'au moment de pointer les " limites du socialisme du XXI e siècle ", on donne déjà aux représentants de ce dernier le bénéfice du doute. On ne fait pas là qu'excuser le pragmatisme de ses héros devenus, au fait de responsables politiques, tributaires de l'efficacité d'un " contrôle citoyen " qui impliquerait un empowerment de masses nécessairement passées par la case école bolivarienne. Plus subrepticement, raisonner en termes de marges de manœuvre de classes gouvernantes dépendantes des circonvolutions d'une économie globale amène aussi à oublier le simple fait qu'elles occupent une certaine position sociale - et non seulement politique - à l'intérieure de celle-ci. Et qu'elles s'affairent avant tout à assurer leurs propres conditions de subsistance et de mobilité sociales, avant celles de leurs administrés, à travers le service quotidien de "la Révolution".

Ce qui se joue alors dans les interactions au sein des espaces contestataires transnationaux, c'est la confirmation (ou l'infirmation) de leur rôle 
de représentants d'une alternative - pour reprendre le prénom originel de l'ALBA, référence explicite à l'adage thatchérien "TINA ${ }^{11}$ ". Tirant parti de l'ambiguité de leur situation socio-spatiale pour se faire passer pour des classes dominées au pouvoir, ces classes dirigeantes escomptent se libérer de tout soupçon de ne faire là que leur travail, au titre de (haut-) fonctionnaires appelés à démultiplier les réseaux de soutien aux régimes qui les font vivre. Leur présence régulière dans ces espaces s'inscrit ainsi dans une économie morale de l'engagement et de ses conditions légitimes d'institutionnalisation, que tout militant avisé devrait bien se garder d'extraire de l'espace-temps ordinaire de l'économie-monde capitaliste. Or il semble que les tenants de l'option "post-altermondialiste " partent trop facilement du principe qu'" ils sont des nôtres ", d'abord en vertu de CV militants qui imposent un respect de rigueur; ensuite parce qu'ils "parlent comme nous ", exposant les mêmes critiques du "système ". Ce notamment à force de lire les mêmes auteurs, dont les modalités d'usage renseignent sur ce qu'ont en commun les néo-bolivariens des deux côtés de l'Atlantique. Ces quelques citations de Chávez à la COP15 l'illustrent assez bien : Rosa Luxembourg - et son bel adage "socialisme ou barbarie! "-, sans pour autant expliquer ce qu'elle dit vraiment des conditions d'un "socialisme démocratique "; l'écrivain uruguayen Eduardo Galleano, auteur du livre culte Les veines ouvertes de l'Amérique latine (1970) - offert à Obama au Sommet des Amériques de 2009 -, pourtant autant critique de l'impérialisme que des élites pro extraversion. Parmi les vivants, un exemple significatif : Hervé Kempf et son livre Comment les riches détruisent la planète (2009), dont le chiffrage de l'horreur de l'exploitation capitaliste mondiale est repris par Chávez juste avant qu'il ne salue celui qui " [le] lui a offert ", "présent dans la salle " - Ignacio Ramonet cofondateur d'Attac et du FSM, directeur du Monde diplomatique et de Mémoire des Luttes. L'esthétique néo-bolivarienne de la subversion des arènes onusiennes, qui passe par un détour sur les lieux a-étatiques que sont les forums sociaux avant de rentrer à nouveau par la grande porte, repose ici sur un double jeu de miroirs : entre des citoyens appelés à "prendre le pouvoir " et ceux qui l'exercent réellement - à l'international - et entre ces derniers et les thuriféraires du "post-altermondialisme ".

Tout phénomène de " circulation internationale des idées " repose sur des stratégies inscrites dans des espaces politiques distincts mais ponctuellement en contact au dehors de leurs frontières ${ }^{12}$ : les " post-altermondialistes " veulent résolument "prendre le pouvoir " en Europe, leurs mentors le garder coûte que coûte. Mais fait rare, ces interactions fonctionnent ici d'autant en vase clos qu'elles se dédoublent d'une " circulation circulaire de l'information ", qui s'opère apparemment tout autant entre les membres du camp pro-ALBA, entrepreneurs à la fois médiatiques et politiques, qu'entre les intellectuels et experts du Quartier latin ${ }^{13}$. Mais tout cela ne serait possible sans la circulation des personnes physiques qui lient ces deux mondes, et dont le carburant n'est autre que cet " excrément du Diable ${ }^{14}$ " qu'est l'or noir. Or du côté des vestiaires comme des loges du spectacle de tribuns aux origines modestes et aux parcours
11. TINA : There is no Alternative.

12. P. Bourdieu, Les conditions sociales de la circulation internationale des idées, Actes de la Recherche en Sciences Sociales, Paris, 2002/5, n 45.

13. P. Bourdieu, Sur la télévision, Raisons d'agir, Paris, 1996.

14. F. Coronil, op. cit. 
15. Que ce statut renvoie d'ailleurs, au sens propre, à des modes de vie " hérités " ou " mérités " - grand bourgeois ou néophytes. Entretiens et observations, Caracas, Paris \& Madrid, entre 2006 et 2012.

16. P. Rimbert, " La pensée critique dans l'enclos universitaire ", Le Monde Diplomatique janvier 2011.

17. www.

larevolucionvive.

org.ve/spip.

php?rubrique20\&lang=es

\& www.

larevolucionvive.

org.ve/spip.

php?article1972\&lang=es

18. J. RANCIÈRE, Le spectateur émancipé, La Fabrique, Paris, 2008.

19. M. Lazzarato, $\mathrm{La}$ fabrique de l'bomme endetté. Essai sur la condition de l'bomme néolibéral, Éditions Amsterdam, Paris, 2011.

20. Entretiens, Caracas, Paris \& Madrid, printemps \& été 2010.

21. P. VAsquez

\& D. FASSIN,

"Humanitarian exception as the rule : the political theology of the 1999 tragedia in Venezuela ", American Ethnologist, 2005/08, $n^{\circ} 32 / 3$ chevaleresques, c'est un tout autre tableau qui se dessine de cette avantgarde. Ses membres peuvent bien arborer les stigmates d'une violence sans nom indistinctement infligée aux révolutionnaires du "Global South ", et qui ne manquent pas de fasciner ses contemporains de la "gauche de la gauche». Il n'empêche que l'élite néo- bolivarienne qui arpente aujourd'hui la scène internationale à grands frais de pétrodollars n'en a pas moins tous les traits d'une nomenklatura du XXI ${ }^{e}$ siècle. C'est-à-dire d'une bourgeoisie d'État, mi-technocrate, mi-intellectuelle, " légaliste " par fidélité aux partis qui assurent sa survie socio-institutionnelle, et " patriote " dans l'exacte mesure de son ancrage cosmopolite ${ }^{15}$. Cet entre-soi est aussi par intermittence l'" autre monde " des " post-altermondialistes ", pour la plupart des travailleurs intellectuels de statut social généralement moindre, mais aspirant eux aussi à "prendre le pouvoir " - beaucoup sont engagés en France au Front de gauche. Ils sont les principaux chefs d'orchestre des campagnes de "solidarité ", "tourisme social " et autres formes de révolutionnarisme par procuration des membres des Cercles bolivariens outre-Atlantique. Désolés du manque d'activisme de recrues potentielles qui préféreraient le " confort " des diplômes universitaires à l'engagement ${ }^{16}$, ils se méfient aussi des jeunes intellectuels par trop " critiques " qui ont renoncé au conseil au Prince en Amérique après y avoir goûté. Dans le "JO " de la partie de cette mouvance qui s'est auto-expatriée à Caracas et investie dans "la réappropriation sociale des ondes ", on peut même lire que "le socialisme bolivarien n'est pas un socialisme de bobos " s'embarrassant de scrupules " anti-totalitaires " "17. C'est dire si dans l'optique d'une militance de gauche, la focale sur la vulgarisation de la critique des médias, comme du spectacle, a le pouvoir de faire renaitre un esprit d'avant-garde, et oublier l'essence proprement matérielle des mécanismes d'exploitation et de domination - et partant, des moyens de les subvertir ${ }^{18}$.

Somme toute, si le néolibéralisme se caractérise bien par un état d'urgence permanent exigeant de dominés passés de la misère à la pauvreté la participation à leur propre exploitation pour rembourser une dette qui n'en finit pas ${ }^{19}$, alors on peut sérieusement douter que l'ALBA en sorte de sitôt. Bien que la dette équatorienne ait pu être allégée grâce à l'audit " citoyen " prôné par le Comité d'annulation de la dette du tiers monde, nombre d'économistes de l'ALBA n'y voient qu'un "répit éphémère dans la lutte désespérée contre un hydre à plusieurs têtes ${ }^{20}$ ". Quant au concept de " dette écologique ", il risque de ne renverser le "système " que sur le plan symbolique. Morales a encore les moyens de ne pas faire à la Pachamama "l'offense de la chiffrer ", mais c'est la logique inverse qui domine le projet Yasuni-ITT - à supposer qu'il vienne un jour à se concrétiser. Dans les trois États indispensables au " commerce des peuples " prôné par l'ALBA, l'intensité des conflits sociaux laisse perplexe quant aux modalités de redistribution et de ré-investissement des rentes minières "socialisées ". Celles-ci relèvent plus à ce stade d'un aggiornamento humanitaire ${ }^{21}$ et traditionaliste de la gouvernementalité néolibérale, que de la marche vers d'hypothétiques lendemains " éco-socialistes ". Le terme peut recou- 
per dans la réalité quelques expériences sensibles en matière de production et de consommation re-localisées et relativement peu polluantes ici et là, mais elles demeurent relativement banales au vu de ce qui a pu se faire ailleurs, au Nord comme au Sud. Ce qui apparaît paradoxal au vu de l'incroyable biodiversité abritée par les territoires de l'ALBA, parmi les mieux dotés au monde en la matière ; mais coule sous le sens du moment que les taux de profits escomptés par les multinationales redevables fiscalement envers ces États - quand elles n'en sont pas partenaires - sont largement supérieurs dans l'économie non " verte ". Au demeurant, l' "écosocialisme " appartient surtout au régime de justification des pouvoirs encourageant à la socialisation des imaginaires des intellectuels de Palais qui l'ont promu.

Du point de vue de la dialectique bolivarienne, l'expression renvoie à l'idéal d'un "âge zéro " d'avant la conquête, parfaitement illustré par la célèbre formule du péruvien Annibal Quijano : "l'Amérique, le capitalisme et les races sont nés le même jour ". En témoigne le détournement par Chávez, en guise de conclusion de son discours de Copenhague, de cette phrase de Simon Bolivar : "si la nature s'y oppose, nous lutterons contre elle et nous ferons en sorte qu'elle nous obéisse ". Devenue un slogan du régime et lisible à ce titre sur des milliers de murs arborant l'espace public vénézuélien, il en subvertit alors radicalement le sens : "si la nature du capitalisme s'y oppose, nous (...) ferons en sorte qu'elle nous obéisse ". De produit d'une civilisation se voulant "maitre et possesseur de la nature ", le Libertador du XIX ${ }^{\mathrm{e}}$ siècle formé au cosmopolitisme grand bourgeois des familles mantuanas ${ }^{22}$ se transforme en précurseur de "l'éco-socialisme " dans l'Amérique "indo-latine " - selon la terminologie à la mode locale. Et malgré l'appétit en matière première ultra-polluante comme en force de travail étasunienne et asiatique du "socialisme ren$\operatorname{tier}^{23}$ " des métropoles côtières, Chávez d'être le fossoyeur du "système métabolique destructif du Capital ". En alimentant, matériellement et symboliquement, la " rente de la dépendance ${ }^{24}$ ", c'est cette utopie d'un " communisme primitif " de "bons sauvages" que permet de faire vivre l'investissement dans la cause du global warming.
22. Élites "créoles" - blancs nés sur place - à l'origine de la Déclaration d'indépendance.

23. L. E. LANDER \& M. LOPEZ-MAyA, El socialismo rentista de Venezuela ante la caida de los precios petroleros, Rosa Luxembourg Foundation, Bruxelles, 2010.

\section{J.-F. BAYART \&}

R. Bertrand, "De quel legs colonial parle-t-on ", Esprit, 2006/12. 
